\section{Clinical \\ complications of \\ Densiron 68 \\ intraocular \\ tamponade for \\ complicated retinal detachment}

W Li', J Zheng, Q Zheng', R Wu'1 X Wang

and $\mathrm{M} \mathrm{Xu}$

\author{
Abstract \\ Purpose To report and disscuss the \\ postoperative complications in patients after \\ Densiron 68 intraocular tamponade in the \\ management of complicated retinal \\ detachment with proliferative \\ vitreoretinopathy (PVR).
}

Methods We presented a prospective interventional non-comparative case series of 27 eyes of 27 consecutive patients. Inclusion criteria were PVR, posterior or inferior retinal breaks, and the patient's inability to posture. Vitreoretinal surgery with Densiron 68 intraocular tamponade was performed in all patients. Complications were recorded at 1 week and 1, 2, and 3 months after Densiron 68 intraocular tamponade and after removal of Densiron 68 endotamponade at same periods. Results The most common complication was posterior capsule opacification and cataract development in seven eyes $\mathbf{2 5 . 9 \%}$ ) and in two eyes $(25 \%, 2 / 8)$, respectively, the second complication was intraocular inflammation in six eyes $(22.2 \%)$, the third complication was emulsification and dispersion and raised intraocular pressure in five eyes (18.5\%), respectively. The success rate with one operation using Densiron 68 was $85.2 \%$ and with further surgery $92.5 \%$. Visual acuity improved from mean $\log M A R$ of 2.12 $(\mathrm{SD}=0.68)$ to $1.16(\mathrm{SD}=\mathbf{0 . 8 4}), P=\mathbf{0 . 0 0 0 1}$. Conclusions According to the results of this study, postoperative complications did not increase significantly in the vitreoretinal surgery with temporary Densiron 68 intraocular tamponade. Densiron 68 intraocular tamponade is safe and effective in treating complicated retinal detachment.
Eye (2010) 24, 21-28; doi:10.1038/eye.2009.57;

published online 27 March 2009

Keywords: complications; silicone oil; vitrectomy; Densiron 68; proliferative vitreoretinopathy; retinal detachment

\section{Introduction}

Repair of rhegmatogenous retinal detachment (RD) using vitrectomy techniques has become increasingly popular. ${ }^{1}$ Inferior RD presents a distinctive surgical challenge to vitreoretinal surgeons, and common vitrectomy with gas tamponade is supplemented with scleral buckling (SB) to achieve adequate tamponade of the inferior retina. However, there has been an increasing trend to move away from SB owing to complications, such as choroidal haemorrhage, ${ }^{2}$ diplopia, ${ }^{3}$ extrusion and infection, ${ }^{4}$ and anterior segment ischaemia. ${ }^{5}$

Silicone oil has been shown to be an effective tamponade agent for complex RD, including those complicated by proliferative vitreoretinopathy (PVR). ${ }^{6}$ It has a lower specific gravity than water, and therefore provides tamponade mainly for the superior retina, especially in the absence of an SB. With the increasing trend to move away from $\mathrm{SB}$, inferior retinal tamponade agents have been developed and used with variable success. For example, intraocular heavy liquids, such as perfluorohexyloctane, have been used to provide postoperative inferior retinal tamponade, ${ }^{7}$ but problems of retinal toxicity ${ }^{8,9}$ and emulsification, ${ }^{7,10}$ owing to lack of viscosity, have been reported. Emulsification in particular
State Key Laboratory Cultivation Base, China National Optometry Center, Eye Hospital, Wenzhou Medical College, Wenzhou, China

Correspondence: W Li, China National Optometry Center,

Eye Hospital, Wenzhou Medical College, 270 Xueyuan Road, Wenzhou 325027,

Zhejiang PR China

Tel: 0577 88068877;

Fax: 057788824115.

E-mail:drlws@

163.net

${ }^{1}$ These authors contributed equally to this work

Received: 14 September 2008

Accepted in revised form: 17 February 2009

Published online: 27 March 2009

The authors have no financial or proprietary interest in any materials or methods described herein. No conflicting relationship exists for any author. 
is thought to contribute to postoperative inflammation and epiretinal membrane (ERM) formation and is thus undesirable. Densiron 68 is a solution of perfluorohexyloctane (F6H8) and $5000 \mathrm{mPas}$ silicone oil, with viscosity $1387 \mathrm{mPas} 3$ and has been promoted for prolonged inferior retinal tamponade, particularly as it has similar physical properties to silicone oil in terms of the shape of the bubble. ${ }^{11}$ The interfacial tension against water at $25^{\circ} \mathrm{C}$ of this solution is $40.82 \mathrm{mN} / \mathrm{m}$. The advantage of this solution is that it increases the viscosity of $\mathrm{F} 6 \mathrm{H} 8$ from 2.5 to $1387 \mathrm{mPa}$, thereby reducing its tendency to disperse (the major reason for the clinical problems that were associated with the use of pure F6H8 as a long-term tamponade). More recently developed heavy tamponades have a higher viscosity, seem to be biologically more stable, are better tolerated, can be left in the eye for a longer period of time, and are associated with fewer complications. ${ }^{11,12}$ However, significant problems have also been reported with the use of these newer substances. ${ }^{13,14}$ In this study, we evaluated deeply postoperative complications in patients after Densiron 68 intraocular tamponade for complicated RD.

\section{Materials and methods}

We performed a prospective interventional noncomparative study of 27 eyes of 27 consecutive patients recruited from the Eye Hospital, Wenzhou Medical College from March 2007 to March 2008. All patients were operated on by the same experienced surgeon (Dr Li).

Standard three-port pars plana vitrectomy was performed in all patients. In the other four patients, there was silicone oil in situ; the oil was removed. Vitrectomy was combined with cataract phacoemulsification and foldable intraocular lens (IOL) implantation in 12 cases. ERMs were peeled in 18 cases. Relaxing retinotomies were performed in 14 patients, and peripheral drainage retinotomies in 9 patients. Perfluorocarbon liquid (PFCL) was used in all cases. PFCL was removed through fluid/air exchange before Densiron 68 was instilled into the eyes. Densiron 68 removal was performed around 1-6 months after the initial surgery, through a pars plana approach using active aspiration through an 18- or 19-gauge needle. Posterior capsulotomies were performed in 11 cases during removal of Densiron 68. One patient who failed with Densiron 68 was given further surgeries involving conventional silicone oil.

The primary study end point was to record any complications arising from surgery, which might be or not be related with the use of Densiron 68. Cases are judged successful when there was re-attachment of the retina in the absence of any tamponade agent. Our secondary end point was to record the visual function and anatomical re-attachment of the retina. Patients were reviewed by the surgeon on 1 day; 1,4 , and 8 weeks; and $3,6,10$, and 15 months with complications and visual acuity. All unscheduled appointments or additional interventions were documented.

\section{Results}

A total of 27 eyes of 27 patients ( 12 men and 15 women) were included in our study. The mean age was 49.2 years $(\mathrm{SD}=19.7)$. Eight eyes $(29.6 \%)$ had earlier RD repair. These included two earlier SB procedures and six vitrectomies. In four cases, conventional silicone oil had been used, in which three cases still had silicone oil in situ at the time of presentation. Gas tamponade was employed earlier in other four cases. At baseline, 4 eyes were aphakic, 20 were phakic, and 3 were pseudophakic, and 3 patients were unable to posture (Table 1). The number of quadrants of RD was $2.74(\mathrm{SD}=0.86)$, and the macula was detached in 25 eyes (92.5\%). The mean duration of Densiron 68 left in situ was 64 days $(\mathrm{SD}=23.2$ days). The mean follow-up was 11.3 months $(\mathrm{SD}=2.3)$.

\section{Anatomical success}

At surgery, complete re-attachment was achieved in all cases. At follow-up, four of 27 eyes had RD (in the superior fundus) with Densiron 68 in situ, and all developed re-detachment after removal of Densiron 68. Two patients were treated successfully with further surgery involving conventional silicone for diffuse PVR in the superior fundus. The other two patients refused to re-operation. No patients developed macular detachment after the application of Densiron 68. At the last followup, the total retinal re-attachment was achieved in 25 of 27 eyes.

\section{Functional success}

Postoperatively, visual acuity improved from mean $\log$ MAR of $2.12(\mathrm{SD}=0.68)$ to a mean of $1.16(\mathrm{SD}=0.84)$. The difference was significant with $P=0.0001$, using a two-tailed paired $t$-test. The preoperative and postoperative visions of individual patients are given in Table 1 . The vision improved in 24 of 27 eyes $(88.8 \%)$. The final $\log$ MAR vision was 1 (Snellen equivalent $6 / 60$ ) or better in $62.9 \%(17 / 27)$; $\log$ MAR of 0.6 (Snellen $6 / 24)$ or better in $18.5 \%$ (5/27), and logMAR of 0.3 (Snellen 6/12) or better in $11.1 \%(3 / 27$, all are $20 / 20)$.

\section{Complications}

The most common complication was cataract development and posterior capsule opacification (PCO) 


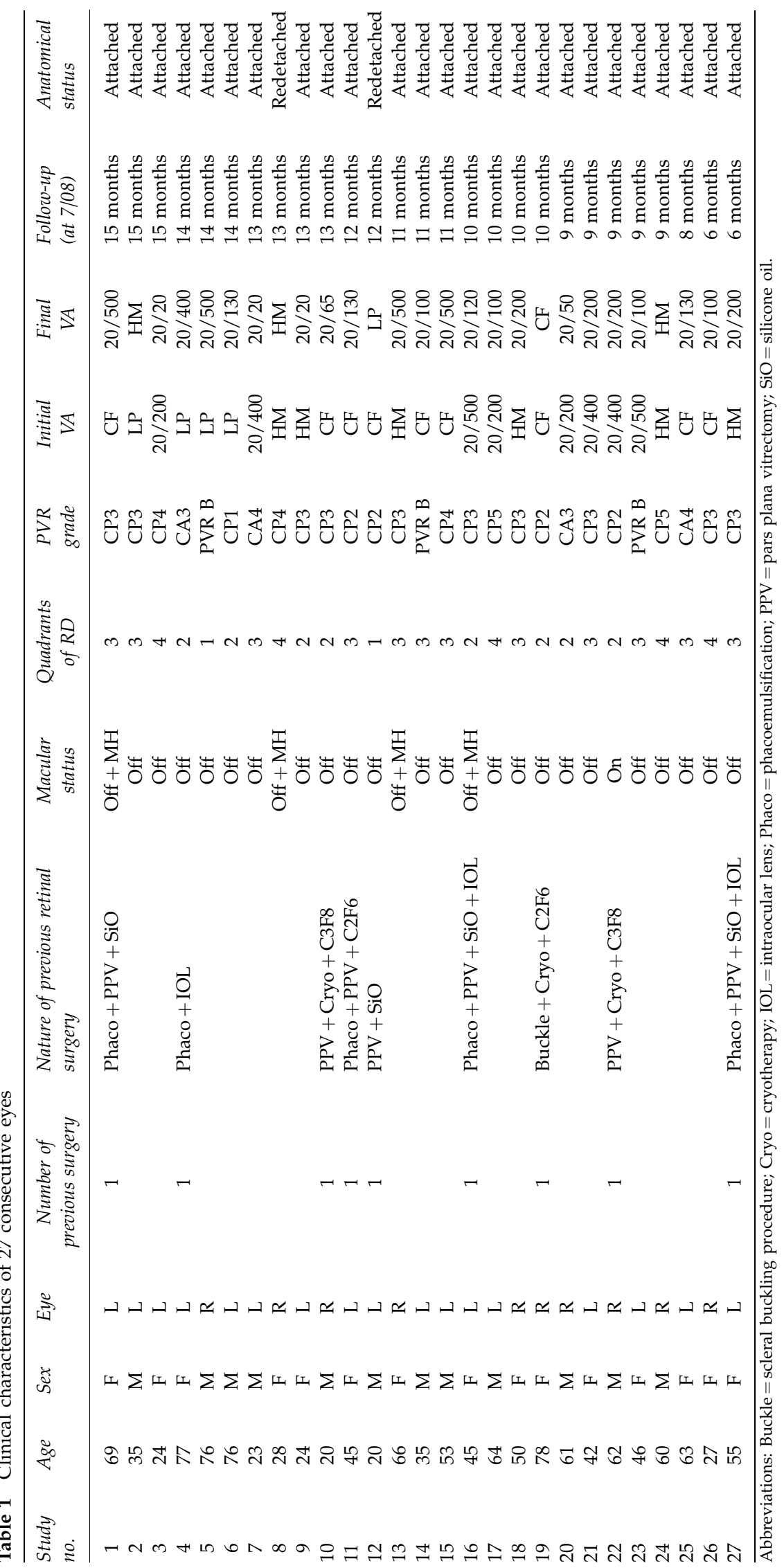


Table 2 Incidence of complications during and after removal of Densiron 68 endotamponade $(n=27)$

\begin{tabular}{|c|c|c|c|c|c|c|c|c|c|c|}
\hline Complications & $\begin{array}{l}\text { 1-week } \\
\text { postop }\end{array}$ & $\begin{array}{l}\text { 1-month } \\
\text { postop }\end{array}$ & $\begin{array}{l}\text { 2-month } \\
\text { postop }\end{array}$ & $\begin{array}{l}\text { 3-month } \\
\text { postop }\end{array}$ & $\begin{array}{c}1 \text {-week } \\
\text { post-ROD }\end{array}$ & $\begin{array}{c}\text { 1-month } \\
\text { post-ROD }\end{array}$ & $\begin{array}{c}\text { 2-month } \\
\text { post-ROD }\end{array}$ & $\begin{array}{c}\text { 3-month } \\
\text { post-ROD }\end{array}$ & $\begin{array}{c}\text { Number } \\
\text { of eyes }\end{array}$ & $\%$ Total \\
\hline Cataract & & 2 & & & & & & & 2 & $25.0(2 / 8)$ \\
\hline Corneal oedema & 2 & & & & & & & & 2 & 7.4 \\
\hline Coroidal detachment & & & & & 3 & & & & 3 & 11.1 \\
\hline Emulsification and dispersion & & 2 & 2 & 1 & & & & & 5 & 18.5 \\
\hline Epiretinal membrane & & 1 & 1 & & & & & & 2 & 7.4 \\
\hline Intraocular haemorrhage & & & & & & 1 & & & 1 & 3.7 \\
\hline Intraocular inflammation & 3 & 2 & & & 1 & & & & 6 & 22.2 \\
\hline Rubeosis iridis & & 1 & & & & & & 1 & 2 & 7.4 \\
\hline Ocular hypotension & & & & & 1 & 1 & 1 & & 3 & 11.1 \\
\hline Posterior capsule opacification & & 4 & 2 & 1 & & & & & 7 & 25.9 \\
\hline Posterior synechiae & & 1 & & & & & & & 1 & 3.7 \\
\hline Pupillary-blocked glaucoma & & 1 & & & & & & & 1 & 3.7 \\
\hline Raised intraocular pressure & 3 & 1 & & & 1 & & & & 5 & 18.5 \\
\hline Re-detachment and PVR & & 2 & & & & 1 & 1 & & 4 & 14.8 \\
\hline Total & 8 & 17 & 5 & 2 & 6 & 3 & 2 & 1 & 44 & \\
\hline
\end{tabular}

Abbreviations: Postop $=$ postoperatively ROD $=$ removal of Densiron 68 .

in nine eyes, the second one was the intraocular inflammation in six eyes, and the third one was the emulsification and dispersion and raised intraocular pressure (IOP) in five eyes, respectively. The number and distribution of complications during and after removal of Densiron 68 endotamponade are presented in Table 2.

\section{Cataract development and PCO}

The most common complication was PCO and cataract development in seven $(25.9 \%)$ and two eyes $(25 \%, 2 / 8)$, respectively. In the 20 phakic eyes at baseline, there were three eyes with pre-existing nuclear cataracts, 5 with early nuclear and 4 with early cortical lens opacities. And 14 eyes of them had their cataracts removed -12 at the time of Densiron 68 injection; 2 at the time of Densiron 68 removal. PCO occurred in seven eyes with Densiron 68 tamponade from 1-month postoperatively to 2 months after removal of Densiron 68. Posterior subcapsular changes were observed in the early postoperative period similar to those observed in conventional silicone oil application, although the happening time were significantly different between them. Capsulotomy was performed during the removal of Densiron 68 in 11 cases.

\section{Intraocular inflammation}

The second complication was intraocular inflammation in six eyes $(22.2 \%)$. A mild-to-moderate inflammatory activity of the anterior segment during Densiron 68 tamponade was observed in 6/27 eyes. Three eyes developed a severe intraocular inflammatory reaction, two of which had fast regression after removal of Densiron 68 and anti-inflammatory therapy (topical steroid application), but the other one eye developed posterior synechiae to the anterior capsulorhexis despite the use of topical steroids for more than 1 month. After removal of Densiron 68, the inflammatory process completely resolved in these eyes.

\section{Raised IOP}

Raised IOP in five eyes (18.5\%) was the third complication. The mean baseline IOP was $8.9 \mathrm{mmHg}$ $(\mathrm{SD}=5.6)$. At 1 week and 1 month after heavy oil operation, five patients presented with an IOP higher than $30 \mathrm{mmHg}$, and at study end, 3 months after oil removal, no patient had raised IOP higher than $20 \mathrm{mmHg}$. At 1 month after heavy oil in vitreous, a papillary-blocked glaucoma was observed and successfully resolved after a neodymium:YAG (Nd:YAG) peripheral iridotomy and treatment with topical steroids. The other cases of IOP increase were successfully treated medically.

\section{Dispersion and emulsification}

Dispersion and emulsification in five eyes (18.5\%) had the same incidence as raised IOP. After heavy oil injection, the oil was found in the anterior chamber in five eyes; in three eyes, remarkable pseudohypopyon (emulsification droplets) was noted in the anterior chamber during the period of Densiron 68 endotamponade. Significant emulsification on the epiretinal surface could not be found. No recurrent emulsification was observed after the routinely performed anterior chamber washout at the time of Densiron 68 removal.

\section{Ocular hypotension}

Three eyes have developed hypotony in our series. All have developed hypotony after removal of Densiron 68 within 1 week; and 1 and 2 months because of recurrent 
RD; two of them subsided after underwent further surgery. The other two patients had extensive anterior PVR and required anterior hyaloid peeling and inferior retinectomies $\left(>270^{\circ}\right)$ before Densiron 68 filling, furthermore, refused to re-operation.

\section{Re-detachment and PVR}

At thee study end point, anatomical failure was due to reproliferation processes with sub-and intraretinal fibrosis and with the formation of new retinal breaks in four eyes (in the superior fundus). There were two cases of ERM due to recurrent RD. Two of them with RD underwent further surgery, with pars plana vitrectomy and by the use of conventional silicone oil. The other two patients refused to re-operation.

\section{Choroidal detachment}

Choroidal detachment happened in three eyes within 1 week after removal of Densiron 68, which subsided after administration of topical and systemic corticosteroids, or both, within 2 weeks after surgery.

\section{Other complications}

Other complications were explained as following: transient corneal oedema was noted in two eyes, which related to elevated IOP, and it resolved within 1 week after surgery as the elevated IOP was controlled; there were two cases of rubeosis iridis due to recurrent RD. In an aphakic eye with intraocular haemorrhage after uncomplicated Densiron 68, removal occurred in the first postoperative month, but it resolved without further intervention within 4 weeks.

\section{Discussion}

Serious and frequent complications have been the major obstacles against the widespread use of heavy tamponades for quite a long time; indeed, some early publications reported considerable complication rates that did not support their use. ${ }^{15,16}$ Many of these severe complications can be associated with an early emulsification and foreign body reaction to the substances used in early series, for example, PFCL and F6H8. ${ }^{10,17}$ Compared with the relative disappointment in these early heavy tamponades, we now seem to have entered into a phase in which we might see the introduction of these vitreous substitutes into routine clinical use. One recently developed heavy tamponade, Densiron 68, has shown better results and fewer complications compared with the earlier used substances, ${ }^{11,12,18}$ and are now slowly gaining acceptance within the vitreoretinal community, despite notable scepticism. ${ }^{12,18,19}$ In this study, the postoperative complications in patients after Densiron 68 intraocular tamponade in the management of complicated RD were investigated in detail.

Cataract development is a known complication of any type of pars plana vitrectomy. Cataract progression has been documented in a high percentage of patients with all types of heavy tamponades. In a multicentre trial and in a comparative study of Densiron 68 , all phakic patients developed some type of cataract progression during the follow-up. ${ }^{18,19}$ In three other series, cataract progression was the frequent postoperative complications of Densiron 68, with an incidence ranging from 8.3 to $25 \% .^{18,19}$ This was similar to the results of our study, in which we reported that the incidence of cataract progression after Densiron 68 tamponade was $25 \%$. The main factors that influence the rate and dynamics of cataract development are the grade of lens opacities at the time of surgery, any type of lens touch that might occur during surgery, the age of the patient, and the tamponade used. No data are available to determine whether cataract surgery should be combined with the initial procedure, with the tamponade removal, or at a later stage. In our department, we currently would leave the crystalline lens in situ during the initial surgery if possible. We then usually combine the removal of the heavy tamponade with cataract surgery and a surgical posterior capsulotomy. Although no comparative data are available, heavy tamponades seem to cause cataract progression and nuclear and posterior subcapsular in a relatively high incidence in patients compared with conventional silicone oil. Opacification of the posterior capsule has been mentioned as a frequent postoperative anterior segment complication in eyes treated with heavy oil tamponade. ${ }^{20}$ We found a relatively high rate of PCO in our series, specifically, in 7 of 27 eyes $(21.5 \%)$ that did not undergo primary capsulectomy at the time of surgery. This could be the result of an increased cellular infiltration, possibly triggered by a foreign body reaction to emulsified tamponade. ${ }^{10,17}$

Some of the early clinical reports of heavy tamponades reported relatively high rates of intraocular inflammations, including fibrin and retropupillary membrane formation. ${ }^{10,16,17}$ In several earlier studies, a mild-to-moderate inflammatory activity of the anterior segment during Densiron 68 tamponade was often observed. In a study, Sandner and Engelmann ${ }^{19}$ reported $21 \%$ mild-to-moderate anterior chamber reactions $(10 / 48)$, but in their another study, mild-to-moderate anterior chamber reactions was i33.3\% (4/12). ${ }^{12}$ Six patients $(22.2 \%)$ in our series had small, segmental synechiae of the iris to the anterior capsule. Topical steroids appeared to be effective in bringing it under good control soon after the surgery. No patients with significant posterior chamber inflammations were seen following the application of a Densiron 68 tamponade in 
our study. The incidence of these complications in our study was comparable to those in other studies in which vitrectomy was combined with Densiron 68 tamponade. However, in contrast to these results, there were a few studies indicated relatively low rates of intraocular inflammations, in which it has been reported that the incidence of intraocular inflammations ranges from 7.1 to $13.1 \% .^{11,21,22}$ All cases could be controlled with topical anti-inflammatory therapy. Majid et $a l^{14}$ reported a single case of fibrinous uveitis associated with Densiron 68. Factors predisposing to fibrin reaction include multiple surgical procedures, such as retinokryopexy, excessive endolaser photocoagulation, large retinotomy, diabetes, and internal tamponade (especially silicone oil). Except for the posterior synechia that occurred in one case, anterior chamber fibrin resolved with topical steroids and non-steroidal anti-inflammatory drugs, and there were no organised fibrin membranes or anterior PVR complications postoperatively.

Elevation of IOP has been observed in clinical series of all heavy tamponades, especially with the first generation of heavy tamponades. ${ }^{23}$ In a comparative series of Wong et al, ${ }^{24}$ the use of Densiron 68 was associated with a higher IOP in the early postoperative period when compared with SO. On day 1, nine eyes $(12.7 \%)$ in the Densiron 68 group and two eyes (3.5\%) in the conventional silicone oil group had IOP higher than $30 \mathrm{mmHg}$. At 4 weeks, IOP $>30 \mathrm{mmHg}$ was seen in nine eyes $(12.7 \%)$ in the Densiron- 68-treated group and in one eye $(1.8 \%)$ in the conventional silicone oil group. The mean IOP was higher in patients treated with Densiron 68 at day 1 and between 7 th and 14th days postoperatively ( $P=0.05$ and 0.01 , respectively). By the 4th week, the IOP difference between the two groups was insignificant $(P=0.17)$. A higher rate of rise in IOP could be noted in $8-30 \%$ patients with Densiron 68 tamponade. ${ }^{11,18,21,22}$ This was similar to the results of our study, in which we reported that the incidence of elevated IOP after Densiron 68 tamponade was $18.5 \%$. At 1 week and 1 month after heavy oil operation, five patients presented with an IOP higher than $30 \mathrm{mmHg}$, and at study end, 3 months after oil removal, no patient had raised IOP higher than $20 \mathrm{mmHg}$. At 1 month after heavy silicone oil in vitreous a pupillary blocked glaucoma was observed, which was successfully resolved by a neodymium: YAG (Nd:YAG) peripheral iridotomy and the topical steroids treatment. But it also has to be kept in mind that an increase in IOP can be seen in a significant number of patients with complicated RDs irrespective of the tamponade agent. ${ }^{25}$ In the vast majority, these can be controlled medically and do not cause a long-term damage. If the rise in IOP is associated with tamponade emulsification, the removal of the tamponade usually leads to a reduction in the IOP. In summary, moderate levels of elevated IOPs can be seen following heavy tamponades. In contrast to conventional oil, it has been seen earlier and more pronounced with the early heavy tamponades, depending on the substance used.

In our study, dispersion and emulsification in five eyes $(18.5 \%)$ was the third complication, starting at 2 weeks postoperatively. After heavy oil injection, the oil was found in the anterior chamber in five eyes. In three eyes, a remarkable pseudohypopyon (emulsification droplets) was noted in the anterior chamber during the period of Densiron 68 endotamponade. Similar results were obtained in large studies involving 99 and 121 cases. $^{18,21}$ With Densiron 68, dispersion occurred in $16(6 / 42)$ and $15 \%(7 / 48),{ }^{11,19}$ remarkable emulsification encountered in $16.7(2 / 12)$ and $10 \%(1 / 10)$ after 1-year follow-up. ${ }^{12,22}$

Only one of the cases described by Sandner and Engelmann ${ }^{19}$ had increased IOP; nevertheless, the authors recommend anterior chamber washout in all patients at the time of Densiron 68 removal. In contrast to these groups, Majid et $a l^{14}$ saw clinically emulsification in 8 out of $40(20 \%)$ cases over a 24-month period. Some of these cases were associated with significant clinical problems (for example, uveitis or ERM formation), others did not seem to be of clinical relevance. We have noted that emulsification and temporary inflammation occur with greater frequency in Densiron 68 patients, it is possible that the lower viscosity of Densiron 68 predisposes to emulsification of oil droplets, which in turn precipitates ocular inflammation, which is consistent with that of Herbrig et $a l^{18}$ and Sandner and Engelmann. ${ }^{19}$ Interestingly, emulsification appears to happen more commonly in younger patients, possibly because of more active lifestyles. The description of emulsification varies from series to series, and sometimes it is subdivided into emulsification visible on clinical examination or during tamponade removal.

In our series, three eyes $(11.1 \%)$ that have developed hypotony. All have developed hypotony after removal of Densiron 68 within 1 week, and 1 and 2 months because of recurrent RD. Ocular hypotension is one of the most feared complications of complex RRD; with conventional tamponades. It can be seen in around $15 \%$ of eyes, and is usually caused by persistent RD, recurrent PVR, and membrane formation over the ciliary body. ${ }^{25}$ It was thought that a foreign body reaction to heavy tamponades triggers an increased inflammatory response, leading to an increased membrane formation with the consequences of re-detachment, recurrent PVR, and cyclitic membranes. In fact, such changes can be seen following unsuccessful surgery with conventional silicone oil, ${ }^{26}$ and similar changes are likely to occur with heavy silicone oil. Whether or not the heavy tamponade, a part of the recently developed heavy silicone oils, adds an additional immunogenic factor is unknown at present. 
However, the combination of the new heavy silicone oils and advances in the surgical technique seem to have led to a lower rate of hypotony over the past years. With Densiron 68, the reported hypotony rates are between 3 and $8.3 \%$ and do not seem to exceed the rate of this complication that would be expected with conventional surgery. ${ }^{12,18,19}$ This was similar to the results in our study. But in contrast to these results, Lim and Vote ${ }^{22}$ reported an incidence of the complication of $20 \%(2 / 10)$ in complex RDs involving inferior PVR by use of Densiron 68 endotamponade, which was much higher than that reported in earlier studies by Herbrig et $a l^{18}(7(3 \%))$, Sandner and Engelmann ${ }^{19}(8(8.3 \%))$, and also higher than that reported in our study $(11.1 \%)$. With the exception of Gerding and Kolck ${ }^{15}$ series, it can therefore be summarised that there is no indication for an increased risk of postoperative hypotony with heavy tamponades compared with conventional tamponades.

Besides PCO, one of the common and visionthreatening postoperative complications in our series was recurrent RD. Four eyes (14.8\%) experienced postoperative RD, which occurred in all cases that had been operated on for RD. Of the four re-detachments, all occurred in association with PVR superiorly, which was found commonly in other studies of F6H8-silicone oil mixtures. ${ }^{11,19}$ Densiron 68 , by virtue of its higher specific gravity, provides better tamponade of the inferior retina than conventional silicone oil, but it appears to do this at the expense of imperfect coverage of the superior retina, possibly predisposing to superior PVR. As with conventional silicone oil tamponades, postoperative retinal re-detachments can occur with a heavy tamponade in situ or after its removal. ${ }^{11,15}$ In contrast to conventional silicone oil, re-detachments with a heavy tamponade in situ usually affect the superior periphery. ${ }^{11,15}$ Only Sandner and Engelmann ${ }^{19}$ found that the majority of redetachments occurred in the lower fundus periphery. In the vast majority of cases, the inferior retina and the posterior pole remain attached with a heavy tamponade in situ. One of the potential advantages of heavy tamponades, compared with conventional oil, might be the lower occurence of macula-off re-detachments, ${ }^{11,27}$ although macula-off detachments have also been described with a heavy tamponade in situ. Most authors think that redetachments following heavy tamponades are easier to treat because they mostly involve the superior retinal quadrants and are more accessible to intraocular gas tamponades than re-detachments of the lower periphery following conventional silicone oil tamponade. The redetachments seen with heavy tamponades in situ in the superior periphery were mainly caused by PVR rather than new breaks or insufficient tamponade of preexisting breaks. ${ }^{11,15}$ ERM formation with an attached retina could also be observed. ${ }^{17}$ Sandner and Engelmann ${ }^{19}$ found that only one in eight redetachments of the superior retina was caused by a new retinal break, although PVR redetachments of the lower periphery have also been described. ${ }^{15}$ Of 10 patients in Lim and Vote $^{22}$ series, 30\% (three eyes) developed recurrent RD during follow-up. In Wong et al ${ }^{11}$ series, recurrent RD occurred in $7.1 \%$ of 42 eyes. ${ }^{11}$ The incidence of these complications in our study was comparable to those in other studies in which vitrectomy was combined with Densiron 68 tamponade.

In summary, it is difficult and often impossible to distinguish between problems caused by the tamponade and those associated with the underlying complicated retinal disease. The complication incidence is also related to the case selection of the individual series, the overall quality of the surgery, the period of time that the tamponade has been left in the eye, the methodology of identifying the complications, and the follow-up period.

Our series has the following limitations. It is uncontrolled, non-comparative, and represents only one surgeon's initial experiences. The rigours of an RCT will best reveal the safety profile of Densiron 68. It is expected that the results of this trial will enable us to better define the current role of the next generation of heavy tamponades.

It seems that heavy silicone oil is a useful tool in selected patients, but not the magic bullet against PVR or a guarantee for good results in every complicated RRD. In summary, our results show that postoperative complications did not increase significantly in the vitreoretinal surgery with temporary Densiron 68 intraocular tamponade. Densiron 68 intraocular tamponade is safe and effective in treating complicated RD.

\section{Acknowledgements}

This study was supported by Wenzhou Medical College of Key Grants (QTJ05025).

\section{References}

1 Ah-Fat FG, Sharma MC, Majid MA, McGalliard JN, Wong $\mathrm{D}$. Trends in vitreoretinal surgery at a tertiary referral centre: 1987 to 1996. Br J Ophthalmol 1999; 83(4): 396-398.

2 Tabandeh H, Sullivan PM, Smahliuk P, Flynn Jr HW, Schiffman J. Suprachoroidal hemorrhage during pars plana vitrectomy. Risk factors and outcomes. Ophthalmology 1999; 106(2): 236-242.

3 Fison PN, Chignell AH. Diplopia after retinal detachment surgery. Br J Ophthalmol 1987; 71(7): 521-525.

4 Flindall RJ, Norton EW, Curtin VT, Gass JD. Reduction of extrusion and infection following episcleral silicone 
implants and cryopexy in retinal detachment surgery. Am J Ophthalmol 1971; 71(4): 835-837.

5 Kwartz J, Charles S, McCormack P, Jackson A, Lavin M. Anterior segment ischaemia following segmental scleral buckling. Br J Ophthalmol 1994; 78(5): 409-410.

6 McCuen II BW, Azen SP, Stern W, Lai MY, Lean JS, Linton $\mathrm{KL}$ et al. Vitrectomy with silicone oil or perfluoropropane gas in eyes with severe proliferative vitreoretinopathy. Silicone Study Report 3. Retina 1993; 13(4): 279-284.

7 Kirchhof B, Wong D, Van Meurs J, Hilgers RD, Macek M, Lois $\mathrm{N}$ et al. Use of perfluorohexyloctane as a long-term internal tamponade agent in complicated retinal detachment surgery. Am J Ophthalmol 2002; 133: 95-101.

8 Chang S, Sparrow JR, Iwamoto T, Gershbein A, Ross R, Ortiz R. Experimental studies of tolerance to intravitreal perfluoro-n-octane liquid. Retina. 1991; 11(4): 367-374.

9 Martinez-Reina MJ, Ruiz-Moreno JM, Montero JA, Rueda J. Histopathology and ultrastructure of rabbit retina after intravitreous injection of perfluorohexyloctane (F6H8). Curr Eye Res 2005; 30(9): 773-779.

10 Hiscott P, Magee RM, Colthurst M, Lois N, Wong D. Clinicopathological correlation of epiretinal membranes and posterior lens opacification following perfluorohexyloctane tamponade. Br J Ophthalmol 2001; 85(2): 179-183.

11 Wong D, Van Meurs JC, Stappler T, Groenewald C, Pearce IA, McGalliard JN et al. A pilot study on the use of a perfluorohexyloctane/silicone oil solution as a heavier than water internal tamponade agent. Br J Ophthalmol 2005; 89(6): 662-665.

12 Sandner D, , Herbrig E, , Engelmann K. High-density silicone oil (Densiron) as a primary intraocular tamponade: 12-month follow up. Graefes Arch Clin Exp Ophthalmol 2007; 245(8): 1097-1105.

13 Theelen T, Tilanus MA, Klevering BJ. Intraocular inflammation following endotamponade with high-density silicone oil. Graefes Arch Clin Exp Ophthalmol 2004; 242(7): 617-620.

14 Majid MA, Hussin HM, Biswas S, Haynes RJ, Mayer EJ, Dick AD. Emulsification of Densiron-68 used in inferior retinal detachment surgery. Eye 2008; 22(1): 152-157.

15 Gerding H, Kolck A. Perfluorohexyloctane as internal tamponade in patients with complicated retinal detachment. Results after 6 months. Ophthalmologe 2004; 101(3): 255-262.

16 Roider J, Hoerauf H, Kobuch K, Gabel VP. Clinical findings on the use of long-term heavy tamponades (semifluorinated alkanes and their oligomers) in complicated retinal detachment surgery. Graefes Arch Clin Exp Ophthalmol 2002; 240(12): 965-971.

17 Vote B, Wheen L, Cluroe A, Teoh H, McGeorge A. Further evidence for proinflammatory nature of perfluorohexyloctane in the eye. Clin Experiment Ophthalmol 2003; 31(5): 408-414.

18 Herbrig E, Sandner D, Engelmann K. Anatomical and functional results of endotamponade with heavy silicone oil-Densiron 68-in complicated retinal detachment. Ophthalmic Res 2007; 39(4): 198-206.

19 Sandner D, Engelmann K. First experiences with highdensity silicone oil (Densiron) as an intraocular tamponade in complex retinal detachment. Graefes Arch Clin Exp Ophthalmol 2006; 244(5): 609-619.

20 Stefaniotou MI, Aspiotis MV, Kitsos GD, Kalogeropoulos CD, Asproudis IC, Psilas KG. Our experience with perfluorohexyloctane ( $\mathrm{F} 6 \mathrm{H} 8)$ as a temporary endotamponade in vitreoretinal surgery. Eur J Ophthalmol 2002; 12(6): 518-522.

21 Stappler T, Heimann H, Wong D, Gibran SK, Groenewald C, Pearce IA. Heavy tamponade 2 Densiron 68(R) in routine clinical practice: anatomical and functional outcomes of a consecutive case series. Eye 2008; 22(10): 1360-1365.

22 Lim BL, Vote B. Densiron intraocular tamponade: a case series. Clin Experiment Ophthalmol 2008; 36(3): 261-264.

23 Bottoni F, Sborgia M, Arpa P, De Casa N, Bertazzi E, Monticelli $\mathrm{M}$ et al. Perfluorocarbon liquids as postoperative short-term vitreous substitutes in complicated retinal detachment. Graefes Arch Clin Exp Ophthalmol 1993; 231(11): 619-628.

24 Wong D, Kumar I, Quah SA, Ali H, Valdeperas X, Romano MR. Comparison of postoperative intraocular pressure in patients with Densiron-68 vs conventional silicone oil: a case-control study. Eye 2007; 23(1): 190-194.

25 Scott IU, Flynn Jr HW, Murray TG, Feuer WJ, Perfluoron study group. Outcomes of surgery for retinal detachment associated with proliferative vitreoretinopathy using perfluoro-n-octane: a multicenter study. Am J Ophthalmol 2003; 136(3): 454-463.

26 Wickham L, Asaria RH, Alexander R, Luthert P, Charteris DG. Immunopathology of intraocular silicone oil: enucleated eyes. Br J Ophthalmol 2007; 91(2): 253-257.

27 Wong D, Cazabon S, Ali H, Kumar I, Valldeperas X, Groenewald $\mathrm{C}$ et al. Can the sequential use of conventional silicone oil and heavy oil be a strategy for the management of proliferative vitreoretinopathy? Ann Acad Med Singapore 2006; 35(3): 181-184. 